\title{
Landmark-based Shape Deformation with Topology-Preserving Constraints
}

\author{
Song Wang \\ Dept. of Computer Science and Engineering \\ University of South Carolina \\ Columbia, SC 29208 \\ songwang@cse.sc.edu
}

\author{
Jim Xiuquan Ji, and Zhi-Pei Liang \\ Dept. of Electrical and Computer Engineering \\ University of Illinois at Urbana-Champaign \\ Urbana, IL 61801 \\ $\{x j i 1, z-l i a n g\} @ u i u c . e d u$
}

\begin{abstract}
This paper presents a novel approach for landmarkbased shape deformation, in which fitting error and shape difference are formulated into a support vector machine (SVM) regression problem. To well describe nonrigid shape deformation, this paper measures the shape difference using a thin-plate spline model. The proposed approach is capable of preserving the topology of the template shape in the deformation. This property is achieved by inserting a set of additional points and imposing a set of linear equality and/or inequality constraints. The underlying optimization problem is solved using a quadratic programming algorithm. The proposed method has been tested using practical data in the context of shape-based image segmentation. Some relevant practical issues, such as missing detected landmarks and selection of the regularization parameter are also briefly discussed.
\end{abstract}

\section{Introduction}

Shape deformation has become a useful tool for many computer vision applications like face recognition and medical image segmentation, where a template shape of the desired structures is deformed to fit some boundary features in a target image. By effectively incorporating some prior shape information of the desired structures into the segmentation process, shape deformation methods are more robust to the image noise and produce more reliable segmentation results.

Since the publication of the active contour (or snakes) model [14], a large group of shape deformation methods $[1,5,24,4]$ have been developed. Those active contour methods usually aim to incorporate some general shape assumptions, like boundary smoothness, into shape deformation. More advanced models are developed to constrain the shape difference between a given template shape and the deformed shape. Jain et al. [13] used a Gaussian distribution of the shape difference to describe the possible template shape deformation, where the shape difference is defined on the trigonometric bases with different frequencies. Zhong et al. [25] applied this technique to segment 3D images by tracking the desired objects slice by slice. Rueckert et al. [18] developed a probabilistic deformable model, where the thin-plate bending energy is used to measure shape difference. Statistical information extracted from multiple template shapes has also been used for shape deformation. Staib et al. [20] represented a shape in the Fourier domain, where the distribution of each Fourier coefficient is learned over a training set. Cootes et al.[6] developed a novel active shape model based on a set of landmarks, in which the joint distribution of those landmarks is statistically learned from a set of training samples using principal component analysis. Along the same line, Leventon et al. [16] incorporated statistical shape information into geodesic active contours for segmentation of medical images.

One important problem in these methods is the preservation of shape topology in the deformation as, in many applications, the shape topology in the target image is known priorly to be the same as that in the template shape. For example, the obtained structures from any $3 \mathrm{D}$ brain image should be consistent with brain anatomy. The methods mentioned above may destroy the shape topology in two different ways. Some of them, like geodesic contour based methods $[4,16]$, explicitly allow the topology inconsistency as they are intentionally designed to do so. In other methods $[14,13,18,20,6]$, the shape topology may be implicitly destroyed in the form of boundary self-intersection.

Because of the importance of shape topology in many computer vision applications involving shape analysis, modelling, and visualization, shape deformation with topology preservation has attracted many researches in recent years. Han et al. [11] generalized the traditional geodesic contour to a topology-preserving version and used it for medical image segmentation. Some topology correction methods, either performed on the meshed surface $[8,9]$ or digital volume $[19,10]$, are also developed in medical imaging applications like brain cortex segmentation. However, most of those methods do not discuss the incorporation of the template shape information.

This paper presents a new landmark-based shape deformation method which can not only incorporate the prior 
template shape information but also preserve the shape topology. We first formulate shape deformation as a support vector machine (SVM) regression problem. To preserve the shape topology, a set of additional points called semilandmarks are then identified and converted into a set of linear equality and/or inequality constraints. The underlying optimization problem is then solved using a quadratic programming algorithm.

The proposed algorithm is described in detail in Section 2. Section 3 presents some experimental results to illustrate its performance, followed by a discussion on some related practical issues (e.g., missing detected landmarks and the regularization parameter selection). Section 4 contains the paper conclusion.

\section{Proposed Method}

According to the statistical shape theory $[15,2]$, a shape instance $\mathbf{V}$ is usually represented by a set of ordered landmarks $\left\{\mathbf{v}_{i} \in \mathbb{R}^{2}, i=1,2, \ldots, n\right\}$ or by a $n$-dimensional column vector $\mathbf{V}=\left(\mathbf{v}_{1}, \mathbf{v}_{2}, \ldots, \mathbf{v}_{n}\right)^{T}$. As illustrated in Fig.1, those landmarks can be treated as an ordered set of sampling points along the desired object boundaries, which can then be approximated using a set of piecewise-linear curves by connecting those landmarks in a given order.
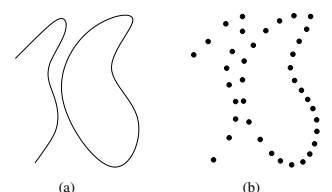

(b)

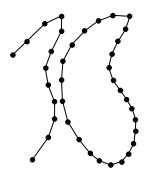

Figure 1. An illustration of a shape instance corresponding to object boundaries. (a) The boundaries of real objects. (b) Corresponding shape instance consisting of sampling landmarks. (c) Approximated boundaries by connecting landmarks as piecewise-linear curves.

The proposed method in essence addresses the following problem: given a template shape $\mathbf{V}=\left(\mathbf{v}_{1}, \mathbf{v}_{2}, \ldots, \mathbf{v}_{n}\right)^{T}$ and a set of corresponding, noisy landmarks $\mathbf{V}^{\prime}=$ $\left(\mathbf{v}_{1}^{\prime}, \mathbf{v}_{2}^{\prime}, \ldots, \mathbf{v}_{n}^{\prime}\right)^{T}$ detected from a target image, deform $\mathbf{V}$ to "best" match $\mathbf{V}^{\prime}$. We formulate the problem as a typical regularization problem in the following form:

$$
\mathbf{U}=\arg \min \left\{\frac{1}{n} \sum_{i=1}^{n} Q\left(\mathbf{u}_{i}, \mathbf{v}_{i}^{\prime}\right)+\lambda d(\mathbf{U}, \mathbf{V})\right\}
$$

where $\mathbf{U}=\left(\mathbf{u}_{1}, \mathbf{u}_{2}, \ldots, \mathbf{u}_{n}\right)^{T}$ is the desired shape, $\lambda$ is the regularization parameter, $Q\left(\mathbf{u}_{i}, \mathbf{v}_{i}^{\prime}\right)$ is the landmark fitting error, and $d(U, V)$ is the shape difference. We next describe the proposed method to solve this problem.

\subsection{Landmark fitting error $Q\left(\mathbf{u}_{i}, \mathbf{v}_{i}^{\prime}\right)$}

A popular loss function $Q(\cdot, \cdot)$ is the squared Euclidean distance

$$
Q\left(\mathbf{u}_{i}, \mathbf{v}_{i}^{\prime}\right)=\left\|\mathbf{u}_{i}-\mathbf{v}_{i}^{\prime}\right\|^{2} \triangleq\left(\hat{x}_{i}-x_{i}^{\prime}\right)^{2}+\left(\hat{y}_{i}-y_{i}^{\prime}\right)^{2},
$$

with $\mathbf{u}_{i}=\left(\hat{x}_{i}, \hat{y}_{i}\right)$ and $\mathbf{v}_{i}^{\prime}=\left(x_{i}^{\prime}, y_{i}^{\prime}\right)$. However, it is well known that the squared loss function is not robust to possible outliers in $\mathbf{V}^{\prime}$. To alleviate this problem, we propose to use the following loss function

$$
Q\left(\mathbf{u}_{i}, \mathbf{v}_{i}^{\prime}\right)=\left\|\mathbf{u}_{i}-\mathbf{v}_{i}^{\prime}\right\|_{\epsilon} \triangleq\left|\hat{x}_{i}-x_{i}^{\prime}\right|_{\epsilon}+\left|\hat{y}_{i}-y_{i}^{\prime}\right|_{\epsilon},
$$

where

$$
|x|_{\epsilon}= \begin{cases}0 & \text { if }|x| \leq \epsilon \\ |x|-\epsilon & \text { else }\end{cases}
$$

is the $\epsilon$-insensitive function proposed by Vapnik [22]. In our implementation of the algorithm, we set $\epsilon=0$. However, the optimization algorithm described in Section 2.4 can also handle the case with non-zero $\epsilon$ if that is desirable.

\subsection{Shape difference measure $d(\mathbf{U}, \mathbf{V})$}

The shape difference between $\mathbf{U}$ and $\mathbf{V}$ is measured by deforming $\mathbf{V}$ to $\mathbf{U}$ using a 2-D thin-plate spline model [3]. This deformation is characterized by $\mathbf{t}=(f, g): \mathbb{R}^{2} \rightarrow \mathbb{R}^{2}$ such that $\mathbf{U}=\mathbf{t}(\mathbf{V})$, i.e., $\mathbf{u}_{i}=\mathbf{t}\left(\mathbf{v}_{i}\right), i=1,2, \ldots, n$, where

$$
\left\{\begin{array}{l}
f(\mathbf{v})=a_{1}+a_{2} x+a_{3} y+\sum_{i=1}^{n} c_{i} K\left(\mathbf{v}, \mathbf{v}_{i}\right) \\
g(\mathbf{v})=b_{1}+b_{2} x+b_{3} y+\sum_{i=1}^{n} d_{i} K\left(\mathbf{v}, \mathbf{v}_{i}\right) .
\end{array}\right.
$$

The parameters $\mathbf{a}=\left(a_{1}, a_{2}, a_{3}\right)^{T}, \mathbf{b}=\left(b_{1}, b_{2}, b_{3}\right)^{T}, \mathbf{c}=$ $\left(c_{1}, c_{2}, \ldots, c_{n}\right)^{T}$, and $\mathbf{d}=\left(d_{1}, d_{2}, \ldots, d_{n}\right)^{T}$ in Eq. (3) can be calculated by solving the following matrix equation:

$$
\left(\begin{array}{cc}
\mathbf{K} & \mathbf{P} \\
\mathbf{P}^{T} & \mathbf{0}
\end{array}\right)\left(\begin{array}{cc}
\mathbf{c} & \mathbf{d} \\
\mathbf{a} & \mathbf{b}
\end{array}\right)=\left(\begin{array}{cc}
\hat{\mathbf{x}} & \hat{\mathbf{y}} \\
\mathbf{0} & \mathbf{0}
\end{array}\right)
$$

where $k_{i j}=K\left(\mathbf{v}_{i}, \mathbf{v}_{j}\right)=\left\|\mathbf{v}_{i}-\mathbf{v}_{j}\right\|^{2} \log \left\|\mathbf{v}_{i}-\mathbf{v}_{j}\right\|$, $i, j=1,2, \ldots, n$, and $\mathbf{P}=(\mathbf{1}, \mathbf{x}, \mathbf{y})$. Note that $\mathbf{x}=\left(x_{1}, x_{2}, \ldots, x_{n}\right)^{T}, \mathbf{y}=\left(y_{1}, y_{2}, \ldots, y_{n}\right)^{T}, \hat{\mathbf{x}}=$ $\left(\hat{x}_{1}, \hat{x}_{2}, \ldots, \hat{x}_{n}\right)^{T}$, and $\hat{\mathbf{y}}=\left(\hat{y}_{1}, \hat{y}_{2}, \ldots, \hat{y}_{n}\right)^{T}$, where $\mathbf{v}_{i}=$ $\left(x_{i}, y_{i}\right)$ and $\mathbf{u}_{i}=\left(\hat{x}_{i}, \hat{y}_{i}\right), i=1, \ldots, n$ are corresponding landmarks between $\mathbf{V}$ and $\mathbf{U}$. It can be shown that the above transform minimizes the following so-called bending energy function [3]

$$
\phi(\mathbf{t})=\iint_{-\infty}^{\infty}(L(f)+L(g)) d x d y,
$$

where $L(\cdot)=\left(\frac{\partial^{2}}{\partial x^{2}}\right)^{2}+2\left(\frac{\partial^{2}}{\partial x \partial y}\right)^{2}+\left(\frac{\partial^{2}}{\partial y^{2}}\right)^{2}$.

Substituting (3) and (4) into (5) yields

$$
\phi(\mathbf{t})=\mathbf{c}^{T} \mathbf{K} \mathbf{c}+\mathbf{d}^{T} \mathbf{K d}=\frac{1}{8 \pi}\left(\hat{\mathbf{x}}^{T} \mathbf{L} \hat{\mathbf{x}}+\hat{\mathbf{y}}^{T} \mathbf{L} \hat{\mathbf{y}}\right),
$$


where $\mathbf{L}$ is the $n \times n$ upper left submatrix of

$$
\left(\begin{array}{cc}
\mathbf{K} & \mathbf{P} \\
\mathbf{P}^{T} & \mathbf{0}
\end{array}\right)^{-1}
$$

$\mathbf{L}$ is only positive semidefinite because the thin-plate bending energy is invariant to affine transforms. If we directly use the bending energy as $d(\mathbf{U}, \mathbf{V})$, the cost function in Eq. (1) may not have a unique optimum. To address this issue, we add another term to account for the landmarkto-landmark squared Euclidean distance between $\mathbf{U}$ and $\mathbf{V}$. Consequently, the proposed shape difference metric is given by

$$
\begin{aligned}
d(\mathbf{U}, \mathbf{V})= & \frac{1}{2}\left\{\hat{\mathbf{x}}^{T} \mathbf{L} \hat{\mathbf{x}}+\hat{\mathbf{y}}^{T} \mathbf{L} \hat{\mathbf{y}}\right\}+\frac{\mu}{2}\left\{(\hat{\mathbf{x}}-\mathbf{x})^{T}(\hat{\mathbf{x}}-\mathbf{x})\right. \\
& \left.+(\hat{\mathbf{y}}-\mathbf{y})^{T}(\hat{\mathbf{y}}-\mathbf{y})\right\},
\end{aligned}
$$

where $\mu$ is a very small positive value (e.g., $\mu=10^{-5}$ ) such that $d(\mathbf{U}, \mathbf{V})$ is dominated by the bending energy except for the degenerated case of an affine transform.

\subsection{Topology preservation constraints}

In many applications, it is desirable to preserve the topology of the template shape after deformation. Consider a shape instance $\mathbf{V}$ consisting of $K$ ordered contours/curves, say $\left\{C_{1}^{v}, C_{2}^{v}, \ldots, C_{K}^{v}\right\}$. Assume that each contour (or curve) $C_{i}^{v}$ is not self-crossed and each pair of contours (or curves) $C_{i}^{v}$ and $C_{j}^{v}$ do not intersect. Then we say another shape instance $\mathbf{U}$ has the same shape topology as $\mathbf{V}$ if and only if

1. U also consists of $K$ ordered contours (or curves) $\left\{C_{1}^{u}, C_{2}^{u}, \ldots, C_{K}^{u}\right\}$.

2. $C_{i}^{u}$ is a closed contour (or open curve) if $C_{i}^{v}$ is a closed contour (or open curve).

3. $C_{i}^{u}, i=1,2, \ldots, K$ is not self-crossed and each pair of contours (or curves) $C_{i}^{u}$ and $C_{j}^{u}$ do not intersect.

4. If $C_{i}^{v}$ is surrounded by a closed contour $C_{j}^{v}, C_{i}^{u}$ is also surrounded by $C_{j}^{u}$ and vice versa.

Some examples of shape instances with destroyed topology are shown in Fig. 2. The paper is focused on avoiding topology destruction caused by boundary crossing shown in Fig. 2(d) and (e) since the case shown in Fig. 2(f) is not likely to happen in real applications.

There are two main reasons that may cause shapetopology destruction in the deformation. The first one comes from the definition of the shape difference measure. To preserve the shape topology, one should choose a shape difference measure $d(\mathbf{U}, \mathbf{V})$ which takes very large value for $\mathbf{U}$ and $\mathbf{V}$ with different topologies. Thin-plate bending

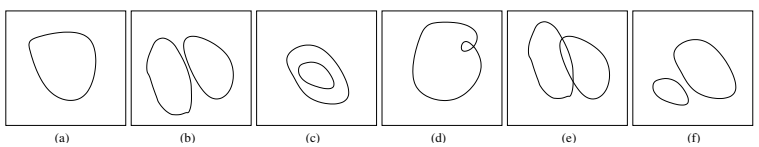

Figure 2. Examples of topology destruction when the template is deformed from (a), (b), and (c) to (d), (e), and (f), respectively.

energy only partially possesses such a property. As illustrated in Fig.3, shape-topology destruction corresponds to the folding of thin plate [3], which usually requires more bending energy. However, this required bending-energy may not be sufficiently large to prevent topology destruction in many applications.

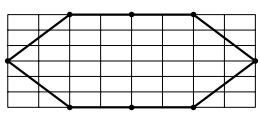

(a)

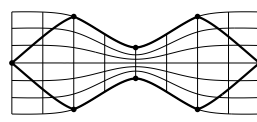

(b)

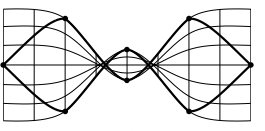

(c)
Figure 3. An illustration of unfolded or folded thin plates and the corresponding bending energy. (a) Template shape, (b) deformed shape with bending energy 0.5076 , and (c) deformed shape with bending energy 2.0303 .

The second reason comes from the boundary approximation with piecewise-linear curves as shape instances in this paper are represented by $n$ ordered landmarks. This approximation may destroy the shape topology even if the thin plate is not folded. An example is shown in Fig.4, where (a) is the template shape consisting of seven landmarks $\{(0,0)$; $(1,0) ;(1,2) ;(2,1.5) ;(3,0) ;(3,2) ;(4,0)\}$. Supposing the deformation transform $\mathbf{t}=(f, g)$ only moves landmark $(2,1.5)$ to $(2,2.5)$, while all the other six landmarks are fixed. The required bending energy $\phi(\mathbf{t})=0.6248$ and the deformed template is shown in Fig.4(b) where the thin plate is not folded. However, when we connect the deformed landmarks using straight line segments (as shown in Fig.4(c)), self-intersections appear and the template shape topology is destroyed.
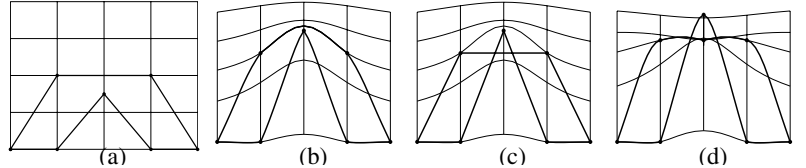

(c)

(d)

Figure 4. An illustration of semilandmark insertion.

If the thin plate is unfolded but the shape topology is destroyed in landmark connection, we know that some line segments between neighboring landmarks in the template shape are in fact deformed into curve segments with large curvature, as shown in Fig.4(c). To address this inconsistency, we can insert "semilandmarks" along those line segments to constrain their deformation. They are called semi- 
landmarks since they are automatically determined by other landmarks and not incorporated into the fitting error. An example is shown in Fig. 4(d) where we insert a semilandmark $(2,2)$ between landmarks $(1,2)$ and $(3,2)$ in the template. The location of this semilandmark is fixed in the deformation process since both landmarks $(1,2)$ and $(3,2)$ are unchanged. Then the thin plate must be folded to obtain such a deformation, whose bending energy is $\phi(\mathbf{t})=1.1584$ that almost doubles the one without inserting a semilandmark as shown in Fig. 4(b).

Based on $n$ landmarks $\left\{\mathbf{v}_{1}, \mathbf{v}_{2}, \ldots, \mathbf{v}_{n}\right\}$, assume $m-n$ $(m \geq n)$ semilandmarks are inserted, which are denoted as $\left\{\mathbf{v}_{n+1}, \ldots, \mathbf{v}_{m}\right\}$. Each semilandmark $\mathbf{v}_{n+k}$ is assumed to lie in a straight line segment connecting two neighboring landmarks $\mathbf{v}_{i_{k}}$ and $\mathbf{v}_{j_{k}}$, i.e.,

$$
\mathbf{v}_{n+k}=p_{k} \cdot \mathbf{v}_{i_{k}}+\left(1-p_{k}\right) \cdot \mathbf{v}_{j_{k}}
$$

where $k=1,2, \ldots, m-n, 1 \leq i_{k}, j_{k} \leq n$ and $0<p_{k}<1$ are known. These introduce a set of topology-preserving constraints that are formulated as

$$
\mathbf{u}_{n+k}=p_{k} \cdot \mathbf{u}_{i_{k}}+\left(1-p_{k}\right) \cdot \mathbf{u}_{j_{k}}
$$

where $\mathbf{u}_{n+k}=\mathbf{t}\left(\mathbf{v}_{n+k}\right)$ is the semilandmark after deformation.

The next step is to introduce more topology-preserving constraints to avoid the folding of thin plates, which cannot be thoroughly addressed by only inserting semilandmarks. The basic principle is illustrated in Fig.5. Boundary selfintersection usually takes place when a landmark point $\mathbf{v}_{i}$ is moved toward and finally get through a line segment $\overline{\mathbf{v}_{s} \mathbf{v}_{t}}$ in the deformation. Therefore, the shape topology can be effectively preserved if we impose an additional constraint to prevent $\mathbf{v}_{i}$ and a semilandmark $\mathbf{v}_{j}$ in $\overline{\mathbf{v}_{s} \mathbf{v}_{t}}$ being moved too close to each other. Projecting the motion of $\mathbf{v}_{i}$ and $\mathbf{v}_{j}$ along $\overline{\mathbf{v}_{i} \mathbf{v}_{j}}$, this paper adopts the constraint

$$
\begin{aligned}
& \frac{\left(\mathbf{u}_{i}-\mathbf{v}_{i}\right) \cdot\left(\mathbf{v}_{j}-\mathbf{v}_{i}\right)}{\left\|\mathbf{v}_{i}-\mathbf{v}_{j}\right\|}+\frac{\left(\mathbf{u}_{j}-\mathbf{v}_{j}\right) \cdot\left(\mathbf{v}_{i}-\mathbf{v}_{j}\right)}{\left\|\mathbf{v}_{i}-\mathbf{v}_{j}\right\|} \\
& \leq\left\|\mathbf{v}_{i}-\mathbf{v}_{j}\right\|-\sigma
\end{aligned}
$$

where $\sigma>0$ is a preselected threshold of the minimum distance (after projected onto $\overline{\mathbf{v}_{i} \mathbf{v}_{j}}$ ) allowed for deformed (semi)landmarks $\mathbf{u}_{i}$ and $\mathbf{u}_{j}$.

\subsection{Proposed deformation algorithm}

Combining the above definitions, the shape deformation problem based on cost function (1) can be written as

$$
\min _{\hat{\mathbf{x}}, \hat{\mathbf{y}}}\left\{\frac{1}{n} \sum_{i=1}^{n}\left(\left|x_{i}^{\prime}-\hat{x}_{i}\right|_{\epsilon}+\left|y_{i}^{\prime}-\hat{y}_{i}\right|_{\epsilon}\right)+\lambda d(\mathbf{U}, \mathbf{V})\right\}
$$

subject to topology-preserving constraints (9) and (10), where $d(\mathbf{U}, \mathbf{V})$ is defined in (7). Note that here $\mathbf{v}_{i}=$

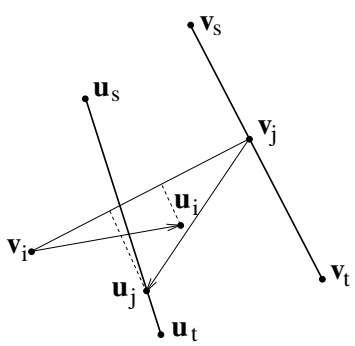

(a)

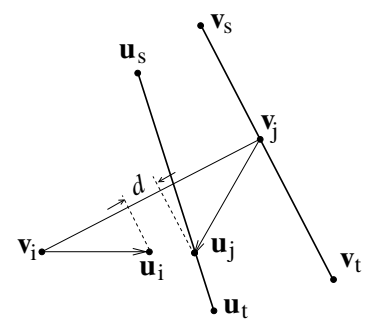

(b)
Figure 5. An illustration of topology-preserving constraints. (a) Deformation of $\mathbf{v}_{i}$ and $\mathbf{v}_{j}$ does not satisfy constraint (10). (b) Deformation of $v_{i}$ and $\mathbf{v}_{j}$ satisfies constraint (10) if the projected distance $d$ is larger than the threshold $\sigma . \mathbf{v}_{j}$ is a semilandmark between neighboring landmarks $\mathbf{v}_{s}$ and $\mathbf{v}_{t}$.

$\left(x_{i}, y_{i}\right)$ and $\mathbf{u}_{i}=\left(\hat{x}_{i}, \hat{y}_{i}\right), i=1,2, \ldots, m$ have been expanded to include both landmarks (for $1 \leq i \leq n$ ) and semilandmarks (for $n+1 \leq i \leq m$ ). $\mathbf{L}$ is the $m \times m$ bending matrix based on all landmarks and semilandmarks, i.e., $\mathbf{v}_{i}, i=1,2, \ldots, m$. After we obtain the optimal $\hat{\mathbf{x}}=\left(\hat{x}_{1}, \hat{x}_{2}, \ldots, \hat{x}_{m}\right)^{T}$ and $\hat{\mathbf{y}}=\left(\hat{y}_{1}, \hat{y}_{2}, \ldots, \hat{y}_{m}\right)^{T}$, we get the deformed shape $\mathbf{U}=\left(\mathbf{u}_{1}, \mathbf{u}_{2}, \ldots, \mathbf{u}_{n}\right)^{T}$ with $\mathbf{u}_{i}=\left(\hat{x}_{i}, \hat{y}_{i}\right)$.

Let $\hat{\mathbf{z}}=\left(\hat{\mathbf{x}}^{T}, \hat{\mathbf{y}}^{T}\right)^{T}$, a $2 m$-dimensional column vector consisting of all the variables to be optimized. Since the constraints (9) and (10) are linear equalities or inequalities with respect to the vector $\hat{\mathbf{z}}$, the above problem can be rewritten as

$\min _{\hat{\mathbf{z}}}\left\{C \sum_{i=1}^{n}\left(\left|x_{i}^{\prime}-\hat{x}_{i}\right|_{\epsilon}+\left|y_{i}^{\prime}-\hat{y}_{i}\right|_{\epsilon}\right)+\frac{1}{2} \hat{\mathbf{z}}^{T} \mathbf{H} \hat{\mathbf{z}}+\mathbf{c}^{T} \hat{\mathbf{z}}\right\}$

subject to

$$
\mathbf{A}^{T} \hat{\mathbf{z}} \leq \mathbf{b}
$$

where $\mathbf{A}_{2 m \times l}$ and $\mathbf{b}_{l \times 1}$ are derived from (9) and (10). Based on (7), it is easy to see that

$$
\mathbf{H}=\left(\begin{array}{cc}
\mathbf{L} & \mathbf{0} \\
\mathbf{0} & \mathbf{L}
\end{array}\right)+\mu \mathbf{I}
$$

is a $2 m \times 2 m$ positive definite matrix and $\mathbf{c}=$ $-\mu\left(\mathbf{x}^{T}, \mathbf{y}^{T}\right)^{T}$ is a known $2 m$-dimensional column vector.

Using the standard SVM technique [22], we can reformulate the problem as follows.

$$
\min _{\hat{\mathbf{z}}, \boldsymbol{\xi}, \hat{\boldsymbol{\xi}}, \boldsymbol{\zeta}, \hat{\boldsymbol{\zeta}}}\left\{C \sum_{i=1}^{n}\left(\xi_{i}+\hat{\xi}_{i}+\zeta_{i}+\hat{\zeta}_{i}\right)+\frac{1}{2} \hat{\mathbf{z}}^{T} \mathbf{H} \hat{\mathbf{z}}+\mathbf{c}^{T} \hat{\mathbf{z}}\right\}
$$


subject to

$$
\begin{array}{cl}
x_{i}^{\prime}-\hat{x}_{i} \leq \epsilon+\hat{\xi}_{i} & i=1,2, \ldots, n \\
\hat{x}_{i}-x_{i}^{\prime} \leq \epsilon+\xi_{i} & i=1,2, \ldots, n \\
y_{i}^{\prime}-\hat{y}_{i} \leq \epsilon+\hat{\zeta}_{i} & i=1,2, \ldots, n \\
\hat{y}_{i}-y_{i}^{\prime} \leq \epsilon+\zeta_{i} & i=1,2, \ldots, n \\
\xi_{i}, \hat{\xi}_{i}, \zeta_{i}, \hat{\zeta}_{i} \geq 0 & i=1,2, \ldots, n \\
\mathbf{A}^{T} \hat{\mathbf{z}} \leq \mathbf{b}, &
\end{array}
$$

where $\boldsymbol{\xi}=\left(\xi_{1}, \xi_{2}, \ldots, \xi_{n}\right)^{T}, \hat{\boldsymbol{\xi}}=\left(\hat{\xi}_{1}, \hat{\xi}_{2}, \ldots, \hat{\xi}_{n}\right)^{T}, \boldsymbol{\zeta}=$ $\left(\zeta_{1}, \zeta_{2}, \ldots, \zeta_{n}\right)^{T}, \hat{\boldsymbol{\zeta}}=\left(\hat{\zeta}_{1}, \hat{\zeta}_{2}, \ldots, \hat{\zeta}_{n}\right)^{T}$, and $C=\frac{1}{n \lambda}$.

Introduce the Lagrange multipliers $\boldsymbol{\alpha}=\left(\alpha_{1}, \alpha_{2}, \ldots\right.$, $\left.\alpha_{n}\right)^{T}, \hat{\boldsymbol{\alpha}}=\left(\hat{\alpha}_{1}, \hat{\alpha}_{2}, \ldots, \hat{\alpha}_{n}\right)^{T}, \boldsymbol{\beta}=\left(\beta_{1}, \beta_{2}, \ldots, \beta_{n}\right)^{T}$, $\hat{\boldsymbol{\beta}}=\left(\hat{\beta}_{1}, \hat{\beta}_{2}, \ldots, \hat{\beta}_{n}\right)^{T}, \quad \gamma=\left(\gamma_{1}, \gamma_{2}, \ldots, \gamma_{n}\right)^{T}$, $\hat{\gamma}=\left(\hat{\gamma}_{1}, \hat{\gamma}_{2}, \ldots, \hat{\gamma}_{n}\right)^{T}, \boldsymbol{\eta}=\left(\eta_{1}, \eta_{2}, \ldots, \eta_{n}\right)^{T}, \hat{\boldsymbol{\eta}}=$ $\left(\hat{\eta}_{1}, \hat{\eta}_{2}, \ldots, \hat{\eta}_{n}\right)^{T}, \boldsymbol{\theta}=\left(\theta_{1}, \theta_{2}, \ldots, \theta_{l}\right)^{T}$. The Lagrangian function for (12) is

$$
\begin{aligned}
& \mathcal{W}(\hat{\mathbf{z}}, \boldsymbol{\xi}, \hat{\boldsymbol{\xi}}, \boldsymbol{\zeta}, \hat{\boldsymbol{\zeta}}, \boldsymbol{\alpha}, \hat{\boldsymbol{\alpha}}, \boldsymbol{\beta}, \hat{\boldsymbol{\beta}}, \boldsymbol{\gamma}, \hat{\boldsymbol{\gamma}}, \boldsymbol{\eta}, \hat{\boldsymbol{\eta}}, \boldsymbol{\theta})= \\
& C \sum_{i=1}^{n}\left(\xi_{i}+\hat{\xi}_{i}+\zeta_{i}+\hat{\zeta}_{i}\right)+\frac{1}{2} \hat{\mathbf{z}}^{T} \mathbf{H} \hat{\mathbf{z}}+\mathbf{c}^{T} \hat{\mathbf{z}} \\
& -\sum_{i=1}^{n} \alpha_{i}\left(\epsilon+\xi_{i}-\hat{x}_{i}+x_{i}^{\prime}\right)-\sum_{i=1}^{n} \hat{\alpha}_{i}\left(\epsilon+\hat{\xi}_{i}+\hat{x}_{i}-x_{i}^{\prime}\right) \\
& -\sum_{i=1}^{n} \beta_{i}\left(\epsilon+\zeta_{i}-\hat{y}_{i}+y_{i}^{\prime}\right)-\sum_{i=1}^{n} \hat{\beta}_{i}\left(\epsilon+\hat{\zeta}_{i}+\hat{y}_{i}-y_{i}^{\prime}\right) \\
& -\sum_{i=1}^{n}\left(\gamma_{i} \xi_{i}+\hat{\gamma}_{i} \hat{\xi}_{i}+\eta_{i} \zeta_{i}+\hat{\eta}_{i} \hat{\zeta}_{i}\right)+(\mathbf{A} \boldsymbol{\theta})^{T} \hat{\mathbf{z}}-\mathbf{b}^{T} \boldsymbol{\theta} .
\end{aligned}
$$

Imposing the first-order necessary conditions (setting the derivatives of the Lagrangian $\mathcal{W}$ with respect to $\gamma, \boldsymbol{\xi}, \hat{\boldsymbol{\xi}}, \boldsymbol{\zeta}$ and $\hat{\zeta}$ to zero) yields

$$
\hat{\mathbf{z}}=\mathbf{H}^{-1}(\mathbf{p}-\mathbf{A} \boldsymbol{\theta}-\mathbf{c})
$$

and

$$
\begin{gathered}
\alpha_{i}+\gamma_{i}=C \\
\hat{\alpha}_{i}+\hat{\gamma}_{i}=C \\
\beta_{i}+\eta_{i}=C \\
\hat{\beta}_{i}+\hat{\eta}_{i}=C \\
\alpha_{i}, \hat{\alpha}_{i}, \beta_{i}, \hat{\beta}_{i}, \gamma_{i}, \hat{\gamma}_{i}, \eta_{i}, \hat{\eta}_{i}, \theta_{j} \geq 0
\end{gathered}
$$

where $i=1,2, \ldots, n, j=1,2, \ldots, l$ and

$$
\mathbf{p}=\left(\hat{\boldsymbol{\alpha}}^{T}-\boldsymbol{\alpha}^{T}, \mathbf{0}_{1 \times(m-n)}, \hat{\boldsymbol{\beta}}^{T}-\boldsymbol{\beta}^{T}, \mathbf{0}_{1 \times(m-n)}\right)^{T} .
$$

Substituting these results into (12) gives the following equivalent formulation:

$$
\begin{aligned}
& \min _{\boldsymbol{\alpha}, \hat{\boldsymbol{\alpha}}, \boldsymbol{\beta}, \hat{\boldsymbol{\beta}}, \boldsymbol{\theta}}\left\{\frac{1}{2}(\mathbf{p}-\mathbf{A} \boldsymbol{\theta}-\mathbf{c})^{T} \mathbf{H}^{-1}(\mathbf{p}-\mathbf{A} \boldsymbol{\theta}-\mathbf{c})\right. \\
& \left.-\mathbf{p}^{T} \mathbf{z}^{\prime}+\mathbf{e}^{T}(\boldsymbol{\alpha}+\hat{\boldsymbol{\alpha}}+\boldsymbol{\beta}+\hat{\boldsymbol{\beta}})+\mathbf{b}^{T} \boldsymbol{\theta}\right\}
\end{aligned}
$$

subject to

$$
0 \leq \alpha_{i}, \hat{\alpha}_{i}, \beta_{i}, \hat{\beta}_{i} \leq C, \quad \theta_{j}>0
$$

where

$$
\begin{aligned}
\mathbf{e} & =(\epsilon, \epsilon, \ldots, \epsilon)^{T} \\
\mathbf{z}^{\prime} & =\left(x_{1}^{\prime}, \ldots, x_{n}^{\prime}, \mathbf{0}_{1 \times(m-n)}, y_{1}^{\prime}, \ldots, y_{n}^{\prime}, \mathbf{0}_{1 \times(m-n)}\right)^{T} .
\end{aligned}
$$

This is a typical quadratic programming problem that can be solved using standard numerical algorithm [22]. After we obtain the optimal values for $\boldsymbol{\alpha}, \hat{\boldsymbol{\alpha}}, \boldsymbol{\beta}, \hat{\boldsymbol{\beta}}$, and $\boldsymbol{\theta}$, the desired $\hat{\mathbf{z}}$ can be calculated from (13).

\section{Experimental Evaluation and Discussion}

This section introduces methods to detect landmark $\mathbf{V}^{\prime}$, presents some representative results to illustrate the performance of the proposed method, and discusses a couple of practical issues.

\subsection{Landmark detection}

Given $\mathbf{V}$, we assume that $\mathbf{v}_{i}^{\prime}$ in an input image falls in a circular area of radius $r_{i}$ centered at $\mathbf{v}_{i}$. According to the principle of anisotropic diffusions [17], non-rigid biologic shape deformation between the template and the input image can be done effectively by moving each landmark $\mathbf{v}_{i}$ along the normal direction of the shape. This means that we can search for the corresponding $\mathbf{V}^{\prime}$ only along the normal directions of $\mathbf{V}$. Denote $\mathbf{n}_{i}$ as the unit vector along the normal direction at $\mathbf{v}_{i}$. Combining the above assumptions, the corresponding landmark $\mathbf{v}_{i}^{\prime}$ can be sought from the line segment $\mathcal{L}_{i}=\left\{\mathbf{v}_{i}+r \mathbf{n}_{i}, r \in\left[-r_{i}, r_{i}\right]\right\}$.

For different application problems, various methods can be used to locate $\mathbf{v}_{i}, i=1,2, \ldots, n$. Generally, they can be categorized into two classes. (a) Edge detection methods. The simplest way is to calculate the gradient vector for each pixel along $\mathcal{L}_{i}$. The point with largest amplitude will be $\mathbf{v}_{i}^{\prime}$. (b) Region matching methods. For any pixel along $\mathcal{L}_{i}$, if its neighboring area has most similar features with the neighboring area centered at $\mathbf{v}_{i}$ in the template image, it will be $\mathbf{v}_{i}^{\prime}$. A number of matching metrics can be used for this problem [21]. Generally, region matching is often more robust than edge detection to process noisy image. However, region matching methods usually require the template landmarks $\mathbf{V}$ to be very accurate, which is not necessary for edge detection methods. In many applications, we may detect multiple candidates for each $\mathbf{v}_{i}^{\prime}$. This problem can be addressed by an iterative algorithm [23], where template shape information is incorporated to choose the best candidate. Another possibility is that no candidate can be detected for some landmarks. This will be discussed in Section 3.3. 


\subsection{Experiments}

In the first example, we applied the proposed algorithm to extract a stria structure from microscopic mice brain images obtained from the Mouse Brain Library. ${ }^{1}$ Figure 6(a) shows a template image and a template shape $\mathbf{V}$ with 19 landmarks manually labelled. Based on the detected landmarks $\mathbf{V}^{\prime}$ shown in Fig. 6(c), we obtained the deformed shape in Fig. 6(d) using the proposed method (without any topology-preserving constraints) with: $C=1.5 \times 10^{-2}$, $\mu=1 \times 10^{-5}, \sigma=5$, and $\epsilon=0$. Note that the thin-plate model provides some resistance to topology destruction (as evident by comparing Fig. 6(d) to Fig.6(c)); The topology is completely preserved using the proposed method (Fig. 6(e)) after imposing a set of topology-preserving constraints as shown in (9) and (10).

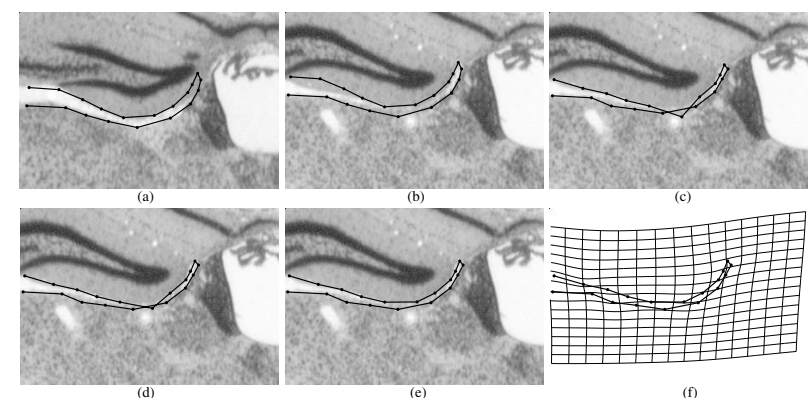

Figure 6. Extraction of a stria structure from microscopic mice brain images: (a) template image and template shape; (b) target image overlaid with the template shape; (c) detected landmarks in the target image; (d) deformed shape without topologypreserving constraints; (e) deformed shape with topology-preserving constraints. (f) deformation field.

In this paper, we choose all the line segments involved in topology destruction (intersect with another nonneighboring line segment as shown in Fig. 6(d)) as $\overline{\mathbf{v}_{s} \mathbf{v}_{t}}$ in (10), where a semilandmark $\mathbf{v}_{j}$ is set halfway between each of those selected line segments. The endpoints of the line segments that intersect $\overline{\mathbf{v}_{s} \mathbf{v}_{t}}$ are then selected as $\mathbf{v}_{i}$ in (10). In addition, to detect landmark $\mathbf{v}_{i}^{\prime}$ as shown in Fig. 6(c), this paper searches pixels with large intensity gradient around $\mathbf{v}_{i}$ in the target image.

In the second example, we applied the proposed method to extract the lateral ventricles from a coronal cryosection brain image. In this example, 28 landmarks were selected to represent the shape, and the parameters used were $C=8 \times 10^{-2}, \mu=1 \times 10^{-5}, \sigma=3$, and $\epsilon=0$. Similar to the first example, the shape topology of the ventricles as defined by the detected landmarks was destroyed (Fig. 7(c)). The deformed shapes without and with topology-preserving

\footnotetext{
${ }^{1}$ http: //www. nervenet. org/mbl .
}

constraints are presented in Fig. 7(d) and (e). Again, the proposed method did a good job in extracting the ventricles.

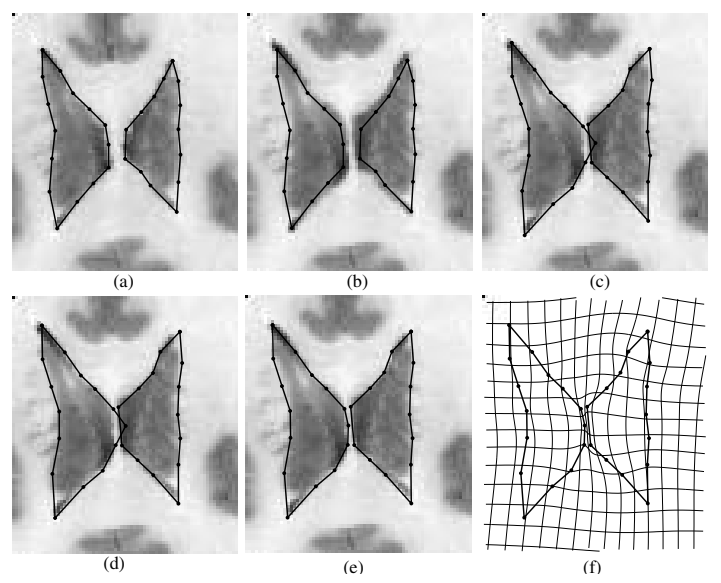

Figure 7. Shape-based segmentation of lateral ventricles in a cryosection image. For (a)-(f), see captions of Fig. 6.

In applying the topology-preserving constraints, we need to check whether the template shape topology has been destroyed after shape deformation and which landmarks are involved. According to the definition and assumption in Section 2.3, destruction of a shape topology implies the intersections of some nonneighboring straight line segments. Detecting such a problem has been well studied [7]. Given two line segments $A B$ and $C D$, they intersect if and only if the endpoints $A$ and $B$ are on opposite sides of the line $C D$, and endpoints $C$ and $D$ are on opposite sides of the line $A B$. The endpoints $A$ and $B$ are on opposite sides of the line $C D$ if and only if

$$
\begin{aligned}
& {\left[\left(a_{y}-c_{y}\right)\left(d_{x}-c_{x}\right)-\left(d_{y}-c_{y}\right)\left(a_{x}-c_{x}\right)\right] .} \\
& {\left[\left(b_{y}-c_{y}\right)\left(d_{x}-c_{x}\right)-\left(d_{y}-c_{y}\right)\left(b_{x}-c_{x}\right)\right]<0,}
\end{aligned}
$$

with $A=\left(a_{x}, a_{y}\right), B=\left(b_{x}, b_{y}\right), C=\left(c_{x}, c_{y}\right)$, and $D=$ $\left(d_{x}, d_{y}\right)$. An efficient "sweepline" algorithm [7] can be used to solve this problem with complexity $O(N \log N)$, where $N$ is the number of line segments.

\subsection{Missing landmarks}

We have so far assumed that a complete set of landmarks $\mathbf{V}^{\prime}=\left(\mathbf{v}_{1}^{\prime}, \mathbf{v}_{2}^{\prime}, \ldots, \mathbf{v}_{n}^{\prime}\right)^{T}$ corresponding to the template landmarks $\mathbf{V}=\left(\mathbf{v}_{1}, \mathbf{v}_{2}, \ldots, \mathbf{v}_{n}\right)^{T}$ are detected in the target image. In practical application of the proposed method, we often need to deal with the scenario of missing detected landmarks because: (a) we may purposely remove some "bad" or unreliable landmarks, and (b) the object may be partly occluded. Let $\tilde{\mathbf{V}}^{\prime}=\left(\mathbf{v}_{1}^{\prime}, \mathbf{v}_{2}^{\prime}, \ldots, \mathbf{v}_{\tilde{n}}^{\prime}\right)^{T}$ be set of the useful landmarks. We can reformulate the minimizing 
problem in (1) as

$$
\frac{1}{n} \sum_{\mathbf{v}_{i}^{\prime} \in \tilde{\mathbf{V}}^{\prime}}\left\|\mathbf{v}_{i}^{\prime}-\mathbf{u}_{i}\right\|_{\epsilon}+\lambda d(\mathbf{U}, \mathbf{V})
$$

subject to the constraints in (9) and (10). It can be shown that this problem can still be solved using same quadratic programming defined by (14) and (15), but with additional constraints:

$$
\alpha_{j}, \hat{\alpha}_{j}, \beta_{j}, \hat{\beta}_{j}=0 ; \quad \forall \mathbf{v}_{j}^{\prime} \notin \tilde{\mathbf{V}}^{\prime} .
$$

While $\mathbf{v}_{j}^{\prime} \notin \tilde{\mathbf{V}}^{\prime}$ is excluded from calculating the fitting error, it is still used in calculating the shape difference, the second item of (16). Alternatively, we may use the algorithm described in Section 2.4 to calculate $\tilde{\mathbf{U}}$ based only on the template landmarks $\tilde{\mathbf{V}}$ corresponding to $\tilde{\mathbf{V}}^{\prime}$. The missing $\mathbf{u}_{j}$ corresponding to $\mathbf{v}_{j} \notin \tilde{\mathbf{V}}$ can be calculated by applying the resulting deformation function $\mathbf{t}=(f, g)$ to $\mathbf{v}_{j}$. However, this formulation prevents the $\mathbf{u}_{j}$ corresponding to missing landmarks from being used in the topologypreserving constraints, which may not be desirable. Otherwise, these two formulations are equivalent.

An example dealing with the case of missing landmarks is shown in Fig. 8. The proposed algorithm is used to extract the corpus callosum contour in a brain image shown in Fig. 8(a), where the template shape consists of 21 landmarks. The parameters used are $C=4 \times 10^{-3}$ and $\mu=1 \times 10^{-6}$. As expected, the missing landmarks (the gaps of the contour shown in Fig. 8(b)) were accurately estimated using the proposed method.

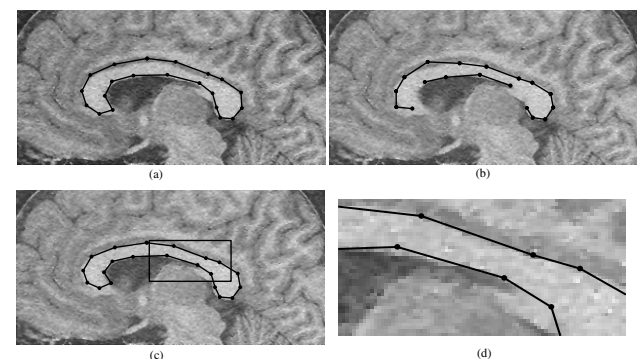

Figure 8. Application of the proposed method to the case of missing landmarks: (a) the target image with the template shape overlaid; (b) detected landmarks on the target image (notice the gaps where landmarks are missing); (c) the deformed shape using the proposed algorithm; (d) the local enlargement of the deformed shape.

\subsection{Selection of the regularization parameter}

Selection of the regularization parameter $\lambda$ or parameter $C$ in (12) is an important problem, which has been well discussed in the literature [12]. In our implementation of the proposed algorithm, we used the L-curve technique [12]. An example is shown in Fig.9, where we tried to extract the boundary of the corpus callosum in an MR brain image. The template shape and the detected landmarks are shown in Fig.9(a) and (b), respectively. Figure 9(e) shows the Lcurve which sequentially connects 12 points corresponding to 12 values of the regularization parameter. The obtained corpus-callosum boundaries using 2 of the values are shown in Figs. 9(c)-(d). As can be seen, the resulting deformed shape is not overly sensitive to the value of the regularization parameter, although it is generally desirable that we select a value corresponds to the corner of the $L$-curve.
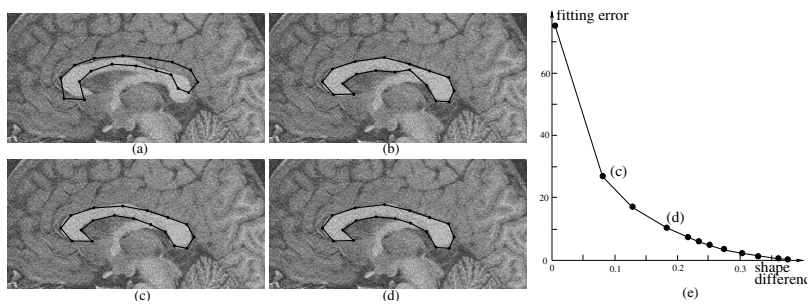

Figure 9. An illustration of selecting regularization parameter using L-curve technique. (a) Target image with the template shape overlaid; (b) detected landmarks; (c-d) deformed shapes with $\lambda$ corresponding to points labelled in the Fig. 9(e); (e) Lcurve approximated by 12 uniformly-sampled regularization parameters.

\subsection{Cardiac sequence tracking}

The last experiment is designed to segment an MR cardiac image sequence. The structures of interest in this experiment are the inner and outer myocardial walls. The accurate segmentation of these structures is a fundamental step to describe and analyze the cardiac function. This image sequence consists of twelve $256 \times 256$ frames, which were acquired in the short-axis plane using a gated cardiac cine method. These frames capture a complete contraction process of the left ventricle, i.e., from the diastole (relaxed) to the systole (contracted). Because of the noise, flow, and data inconsistency during gated image data collection, the boundaries of the myocardial walls are blurred.

As shown in the top-left of Fig. 10, the first frame is manually segmented to construct a template shape that consists of two circular contours. The inner and outer contours consist of 33 and 35 uniformly distributed landmarks (red dots), respectively. In this figure, only a $130 \times 100$ portion around the heart is displayed for better visual effect. The resulting segmentation is shown in Fig. 10 in the time order from the top-left to the bottom-right.

\subsection{Other related issues}

One may desire the nonrigid biologic shape to deform only along the normal direction. This may help reduce the 


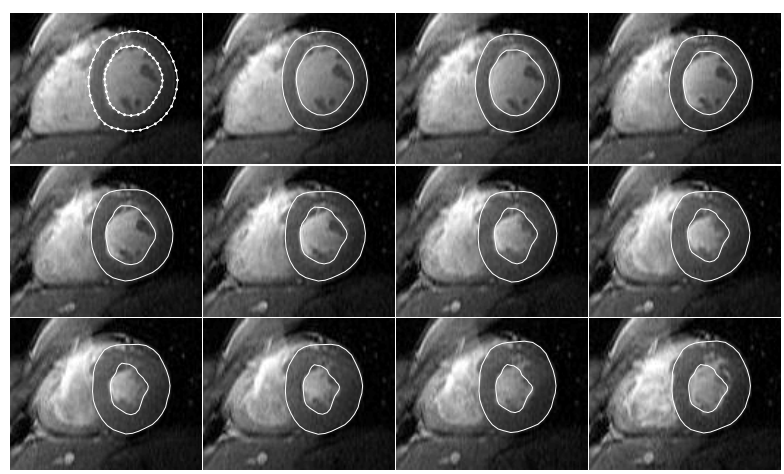

Figure 10. Segment myocardial walls from an MR cardiac image sequence.

chance of moving two neighboring landmarks too close and destroying the shape topology. Let $\mathbf{n}_{i}=\left(N_{i x}, N_{i y}\right)$ be the normal direction of the template shape $\mathbf{V}$ at landmark $\mathbf{v}_{i}$. The line connecting $\mathbf{u}_{i}$ to $\mathbf{v}_{i}$ should be in the same direction as $\mathbf{n}_{i}$. This can be written as

$$
N_{i x} \cdot\left(\hat{y}_{i}-y_{i}\right)=N_{i y} \cdot\left(\hat{x}_{i}-x_{i}\right), i=1,2, \ldots, n,
$$

which are also linear equality constraints on $\hat{\mathbf{z}}$ and the corresponding deformation can be found using the same quadratic-programming algorithm.

\section{Conclusion}

This paper presented a new method for thin-plate splinebased shape deformation in the presence of noisy landmarks. The shape deformation problem is formulated as a regularization problem using an $\epsilon$-insensitive loss function to measure the fitting error of landmarks and the thin-plate bending energy to measure shape difference. Specific linear constraints are introduced to effectively preserve the shape topology in the deformation. The underlying optimization problem is solved using a quadratic programming algorithm. The proposed method can be used for shape-based image segmentation and object tracking in spatial/temporal image sequences.

Acknowledgement This research is supported, in part, by an NSF-ITR grant titled "Shape Exploration for Medical Applications - From Representation, Correspondence, Deformation to Image Segmentation."

\section{References}

[1] A. A. Amini, S. Tehrani, and T. E. Weymouth. Using dynamic programming for minimizing the energy of active contours in the presence of hard constraints. In Proc. ICCV, pages 95-99, 1988.

[2] F. L. Bookstein. The Measurement of Biological Shape and Shape Change. Lecture Notes in Biomathematics 24. Springer-Verlag, New York, 1978.

[3] F. L. Bookstein. Principal warps: Thin-plate splines and the decomposition of deformations. IEEE Trans. PAMI, 11(6):567-585, June 1989.
[4] V. Casselles, F. Catte, T. Coll, and F. Dibos. A geometric model for active contours. Numerische Mathematik, 66(1):1-31, Jan. 1993.

[5] L. D. Cohen and I. Cohen. A finite element method applied to new active contour models and $3 \mathrm{~d}$ reconstruction from cross sections. In Proc. ICCV, pages 587-591, 1990.

[6] T. F. Cootes, C. J. Taylor, D. H. Cooper, and J. Graham. Active shape models - their training and application. CVIU, 61(1):38-59, Jan. 1995.

[7] T. Cormen, C. Leiserson, and R. Rivest. Introduction to Algorithms. MIT Press, Cambridge, MA, 1990.

[8] B. Fischl, A. Liu, and A. M. Dale. Automated manifold surgery: Constructing geometrically accurate and topologically correct models of the human cerebral cortex. IEEE Trans. Med. Imag., 20(1):70-80, January 2001.

[9] I. Guskov and Z. J. Wood. Topological noise removal. In Proc. Graphics Interface, pages 19-26, 2001.

[10] X. Han, U. Braga-Neto, and J. L. Prince. Topology correction in brain cortex segmentation using a multiscale, graphbased algorithm. IEEE Trans. Med. Imag., 21(2):109-121, 2002.

[11] X. Han, C. Xu, and J. L. Prince. A topology preserving deformable model using level set. In Proc. IEEE CVPR, volume 2, pages 765-770, 2001.

[12] P. C. Hansen. Analysis of discrete ill-posed problems by means of the L-curve. SIAM Review, 34(4):561-580, 1992.

[13] A. K. Jain, Y. Zhong, and S. Lakshmanan. Object matching using deformable templates. IEEE Trans. PAMI, 18(3):267278, March 1996.

[14] M. Kass, A. Witkin, and D. Terzopoulos. Snakes: Active contour models. IJCV, 1(4):321-331, 1987.

[15] D. G. Kendall, D. Barden, T. K. Carne, and H. Le. Shape and Shape Theory. John Wiley \& Sons, LTD, 1999.

[16] M. E. Leventon, E. L. Grimson, and O. Faugeras. Statistical shape influence in geodesic active contours. In Proc. IEEE CVPR, pages 316-323, 2000.

[17] P. Perona, T. Shiota, and J. Malik. Anisotropic diffusion. In Geometry-Driven Diffusion in Computer Vision, pages 7392. Dordrecht: Kluwer, 1994.

[18] D. Rueckert and P. Burger. Geometrically deformable templates for shape-based segmentation and tracking in cardiac MR image. In Proc. EMMCVPR, pages 83-98, 1997.

[19] D. W. Shattuck and R. M. Leahy. Topologically constrained cortical surfaces from MRI. In Proc. SPIE, volume 3979 , pages 747-758, 2000.

[20] L. H. Staib and J. S. Ducan. Boundary finding with parametrically deformable models. IEEE Trans. PAMI, 17(11):1061-1075, Nov. 1992.

[21] A. W. Toga. Brain Warping. Academic Press, 1999.

[22] V. N. Vapnik. The Nature of Statistical Learning Theory. New York: Springer-Verlag, 2000.

[23] S. Wang, W. Zhu, and Z.-P. Liang. Shape deformation: SVM regression and application to medical image segmentation. In Proc. ICCV, pages (II)209-216, 2001.

[24] D. J. Williams and M. Shah. A fast algorithm for active contours. In Proc. ICCV, pages 592-595, 1990.

[25] Y. Zhong, A. K. Jain, and M. P. Dubuisson-Jolly. Object tracking using deformable template. IEEE Trans. PAMI, 22(5):544-549, May 2000. 